\title{
Síntese pelo método da coprecipitação e caracterização estrutural do tungstato de cálcio com estrutura tipo scheelita
}

\section{(Synthesis by the coprecipitation method and structural characterization of calcium tungstate with scheelite type structure)}

\author{
E. G. Vieira, P. A. A. Sousa, J. M. E. Matos, M. R. M. C. Santos \\ Laboratório Interdisciplinar de Materiais Avançados - LIMAV, Centro de Ciências da Natureza - CCN, \\ Universidade Federal do Piauí - UFPI, Campus Universitário Ministro Petrônio Portella, Bairro Ininga, \\ Teresina, PI 64049-550 \\ jmematos@ufpi.edu.br
}

\begin{abstract}
Resumo
Nanocristais de $\mathrm{CaWO}_{4}$ foram sintetizados pelos métodos de coprecipitação com diferentes razões do solvente (água/etilenoglicol) e processados à temperatura ambiente. Estes nanocristais foram caracterizados por difração de raios X, espectroscopia Raman, espectroscopia na região do infravermelho com transformada de Fourier (FT-IR) e espectrofotometria de absorção na região ultravioleta-visível. De acordo com os padrões de raios $\mathrm{X}$, os espectros dos cristais exibiram apenas a estrutura tetragonal do tipo scheelita sem a presença de fases intermediárias. Os dados obtidos pelos refinamentos Rietveld revelaram que os átomos de oxigênio ocupam posições diferentes nos "clusters" do tipo $\left[\mathrm{WO}_{4}\right]$ sugerindo a presença de distorções nesses aglomerados. Os espectros de Raman mostraram dez modos vibracionais ativos $\left(2 \mathrm{~A}_{\mathrm{g}}, 5 \mathrm{~B}_{\mathrm{g}}\right.$ e $\left.3 \mathrm{E}_{\mathrm{g}}\right)$ para o óxido, os espectros de FT-IR exibiram uma banda de absorção larga situada entre $796 \mathrm{~cm}^{-1}$ a $801 \mathrm{~cm}^{-1}$ que está associado a vibrações antissimétricas na ligações W-O dentro dos "clusters" de [ $\mathrm{WO}_{4}$ ] e duas bandas nas regiões de $436 \mathrm{~cm}^{-1}$ a $437 \mathrm{~cm}^{-1}$ relacionados com vibrações de flexão. As medições de absorção ultravioleta-visível evidenciou a variação dos valores da "band gap" ópticana faixa de 5,35 a 5,39 eV.

Palavras-chave: tungstato de cálcio, scheelite, coprecipitação.
\end{abstract}

Abstract

$\mathrm{CaWO}_{4}$ nanocrystals were synthesized by the coprecipitation method of with different solvent ratio (water/ethylene glycol) and processed at room temperature. These nanocrystals were characterized by X-ray diffraction (XRD), Raman spectroscopies, Fourier transform infrared (FT-IR) e ultraviolet-visible (UV-Vis) absorption spectroscopies. According to the X-ray patterns, of all the crystals exhibited only a tetragonal scheelita structure without the presence of deleterious phases. The data obtained by the Rietveld refinements revealed that the oxygen atoms occupy different positions in the $\left[\mathrm{WO}_{4}\right]$ "clusters", suggesting the presence of lattice distortions. The Raman spectra showed ten active vibrational modes $\left(2 A_{g}, 5 B_{g}\right.$ e $\left.3 E_{\mathrm{g}}\right)$ for the oxide, the FT-IR spectra exhibited a large absorption band situated at around in the regions of $796 \mathrm{~cm}^{-1}$ to $801 \mathrm{~cm}^{-1}$, which is associated to the W-O antisymmetric stretching vibrations into the [WO $]$ "clusters" and two bands in the regions of $436 \mathrm{~cm}^{-1}$ to $437 \mathrm{~cm}^{-1}$ related to bending vibrations. The ultraviolet-visible absorption measurements evidenced the variation of optical band gap values in range from 5.35 to $5.39 \mathrm{eV}$.

Keywords: calcium tungstate, scheelite, coprecipitation.

\section{INTRODUÇÃO}

O estudo e o desenvolvimento de determinados materiais semicondutores, com propriedades ópticas ativas como, por exemplo, propriedades fotoluminescentes, propriedades fotocatalíticas, eletroluminescência, propriedades ópticas não lineares vem sendo estudado diariamente pela comunidade científica $[1,2]$. Os molibdatos $\left(\mathrm{MoO}_{4}^{2-}\right)$ e os tungstatos $\left(\mathrm{WO}_{4}{ }^{2-}\right)$, em especial o tungstato de cálcio $\left(\mathrm{CaWO}_{4}\right)$, são exemplos desses materiais. Esses materiais inorgânicos fotoluminescentes têm aplicações tecnológicas em quase todos os dispositivos que possa a vir produzir luz artificial e em processos catalíticos [3, 4]. Dentre essas aplicações podem-se destacar as seguintes: produção de lasers de estado sólido, produção de diodos emissores de luz (LEDs) e a sua utilização em processos fotocatalíticos heterogêneos para fotodegradação de corantes $[5,6]$. Os tungstatos $\left(\mathrm{WO}_{4}^{2-}\right)$ de alcalinos terrosos apresentam ampla variedade de aplicações por possuírem alta estabilidade térmica, além de eficiente absorção de radiações de alta energia (UV, raios $\mathrm{X}$, etc.) [7, 8]. As propriedades dos tungstatos que despertam maior atenção dos pesquisadores é a fotoluminescência e fotocatálise. Nesse sentido, muitos estudos investigando a resposta óptica no visível, ultravioleta próximo e regiões do infravermelho tem sido realizados [9].

Os materiais luminescentes conhecidos como "fósforos" são sólidos que convertem certo tipo de energia em radiação eletromagnética nas regiões do ultravioleta, visível e 
infravermelho. Esses materiais consistem de uma rede hospedeira inerte e um ativador opticamente excitado como, por exemplo, metais de transição $(3 d)$ ou lantanídeos (4f) $[9,10]$.

O nome scheelita é utilizado para descrever a estrutura cristalina comum da classe dos materiais $\mathrm{MWO}_{4}(\mathrm{M}=\mathrm{Ca}$, $\mathrm{Sr}, \mathrm{Ba}, \mathrm{Pb}$ ). O $\mathrm{CaWO}_{4}$ do tipo scheelita, possui estrutura cristalina tetragonal grupo espacial I41/a e grupo de simetria $\left(\mathrm{C}_{4 \mathrm{~h}}^{6}\right)$, sendo que o cátion $\mathrm{Ca}^{2+}$ apresenta-se coordenado a 8 íns de oxigênio e o cátion de $\mathrm{W}$ apresenta-se coordenado a 4 íons de oxigênio, de modo que a célula unitária apresenta duas unidades $\mathrm{CaWO}_{4}[11,12]$.

As propriedades físico-químicas desses sólidos inorgânicos dependem exclusivamente das suas estruturas, da distribuição de composição, da sua pureza, da fase cristalina e de seu tamanho. Neste ponto de vista, o método da coprecipitação pode ser considerado excelente candidato para este fim. O processo de precipitação deverá ser quantitativo e simultâneo, sem que ocorra a separação preferencial de alguns dos constituintes nos precipitados formados. Em alguns casos, é necessário um controle rígido de parâmetros como o pH da solução, viscosidade, temperatura, concentração dos reagentes e controle na diferenças de solubilidade entre as várias fases precipitantes que afetam fortemente a cinética de precipitação, para a obtenção de pós com as características adequadas [13, 14].

$\mathrm{O}$ aumento da proporção mássica de solventes não aquosos como, por exemplo, o etileno glicol usado como solvente na síntese/processamento pode inibir o processo de crescimento dos agregados, levando a uma modificação em seus tamanhos/morfologia e possíveis distorções nos "clusters" podem influenciar na fase cristalina como também na máxima emissão fotoluminescente das partículas [15].

Portanto, neste trabalho estudou-se a síntese de nanocristais de $\mathrm{CaWO}_{4}$ do tipo scheelita pelo método da coprecipitação (MCP) visando o estudo da influência da variação das proporções do solvente não-aquoso etileno glicol na formação da sua fase cristalina para um futuro estudo das suas propriedades fotoluminescentes (FL).

\section{EXPERIMENTAL}

\section{Procedimentos de síntese}

Para a síntese do tungstato de cálcio foram utilizadas as rotas sintéticas do MCP. O composto foi sintetizado a partir do nitrato do metal alcalino terroso $\mathrm{Ca}\left(\mathrm{NO}_{3}\right)_{2} \cdot 4 \mathrm{H}_{2} \mathrm{O}$ (99\% de pureza, Isofar), o tungstato de sódio bihidratado $\left(\mathrm{Na}_{2} \mathrm{WO}_{4} \cdot 2 \mathrm{H}_{2} \mathrm{O}\right)$ (99\% de pureza, Vetec), o solvente nãoaquoso etilenoglicol (99,5\% de pureza, Vetec) e água deionizada. Os precursores foram estequiometricamente pesados na proporção de 1:1, ou seja, foram utilizados 5,0 mmolar do nitrato de cálcio e do tungstato de sódio e solubilizados em água deionizada e em etilenoglicol. Assim, foram dissolvidos em uma mistura contendo um total de 100 $\mathrm{g}$ de diferentes proporções em massa de solventes $\left(\mathrm{xH}_{2} \mathrm{O}: 1\right.$ $\mathrm{xC}_{2} \mathrm{H}_{6} \mathrm{O}_{2}$ ), onde $\mathrm{x}=1 ; 0,75 ; 0,50 ; 0,25$ e 0 . Na reaçã̃o de precipitação, os cátions $\mathrm{Ca}^{2+}$ são receptores de elétrons (ácidos de Lewis), enquanto os ânions $\mathrm{WO}_{4}^{2-}$ são doadores de elétrons (bases de Lewis). As soluções com diferentes proporções de solventes (água/etileno glicol), os quais têm alta afinidade e miscibilidade devido à formação de ligações de hidrogênio entre si não sofreram ajuste no seu pH com um bom rendimento reacional. Utilizando-se das proporções dos solventes anteriormente citadas, a síntese do $\mathrm{CaWO}_{4}$ ocorreu em temperatura ambiente, sob agitação, durante aproximadamente $30 \mathrm{~min}$. Após a reação, as soluções resultantes foram centrifugadas, lavadas sucessivamente com água deionizada e acetona e o produto seco em estufa a $80^{\circ} \mathrm{C}$.

\section{Caracterização do material}

Os materiais sintetizados neste trabalho foram caracterizados por difração de raios X (DRX) em um equipamento Shimadzu Labx-XRD 6000, radiação $\mathrm{Cu}-\mathrm{k} \alpha(\lambda$ $=1,5406 \AA$ ) e monocromador de grafite. Os ensaios foram realizados no intervalo de $5^{\circ}$ a $75^{\circ}$, com taxa de varredura de $2^{\circ} \mathrm{min}^{-1}$ e tempo total de exposição de $35 \mathrm{~min}$. Com o auxílio do software X'Pert HighScore Plus (Versão 2.0.1, Windows), os difratogramas obtidos foram comparados com perfis reportados na literatura e seus respectivos arquivos da base de dados Inorganic Crystal Structure Database (ICSD). Para o refinamento de Rietveld, os ensaios foram realizados no intervalo de $5^{\circ}$ a $75^{\circ}$, com taxa de varredura de $0,02^{\circ}$ $\mathrm{min}^{-1}$ com o tempo total de exposição de $90 \mathrm{~min}$. O software DBWSTool (Versão 2.3 Windows) foi utilizado para calcular parâmetros de rede experimentais. O estudo e representação das células unitárias dos óxidos foram feitos utilizandose o software Diamond Crystal and Molecular Structure Visualization (Versão 3.2e para Windows), juntamente com os parâmetros de rede, posições atômicas obtidas por meio do refinamento de Rietveld.

A espectroscopia Raman foi feita em um equipamento de Micro-Raman Confocal Bruker Senterra, com microscópio Olympus BX50, monocromador ligado a um dispositivo de carga acoplada (CCD - "Charge-Coupled Device") e, ajuste automático da resolução em $0,1 \mathrm{~cm}^{-1}$ na região espectral de $50 \mathrm{a} 1100 \mathrm{~cm}^{-1}$. As amostras foram analisadas à temperatura ambiente, utilizando um laser de $532 \mathrm{~nm}$ com uma potência de saída de $10 \mathrm{~mW}$, com um tempo de integração de $10 \mathrm{~s}$.

A análise vibracional na região do infravermelho foi feita em um equipamento Bruker Vertex 70 na região espectral de 400 a $1300 \mathrm{~cm}^{-1}$. Os espectros foram obtidos no modo de transmissão com um total de 64 varreduras e uma resolução espectral de $4 \mathrm{~cm}^{-1}$. A técnica de pastilhas de $\mathrm{KBr}$ foi empregada no preparo das amostras, onde uma alíquota de material foi previamente adicionada ao $\mathrm{KBr}$ e posteriormente homogeneizada com almofariz e pistilo, seguida de prensagem. Ao término desta etapa, as pastilhas foram armazenadas em dessecador durante alguns minutos, com a finalidade de remover o excesso de água adsorvida durante a preparação.

Para as análises dos pós por espectroscopia na região do 
ultravioleta-visível (UV-Vis) foi utilizado um equipamento Varian Cary 300, no modo refletância difusa com comprimento de onda na faixa de 190 a $400 \mathrm{~nm}$. Utilizouse óxido de magnésio $(\mathrm{MgO})$ como material de referência e um porta amostra especial para pós foi adaptado ao sistema. A partir dos espectros de refletância, determinados à temperatura ambiente, foi possível estabelecer a energia de "gap" desses materiais.

\section{RESULTADOS E DISCUSSÃO}

Para elucidar o efeito das diferentes proporções dos solventes $\left(\mathrm{H}_{2} \mathrm{O} / \mathrm{C}_{2} \mathrm{H}_{6} \mathrm{O}_{2}\right)$ durante a síntese pelo método da coprecipitação, os pós de $\mathrm{CaWO}_{4}$ foram analisados pela técnica de DRX. A Fig. 1 mostra o difratograma de raios $\mathrm{X}$ para as diferentes proporções mássicas do solvente etileno glicol. Fases cristalinas intermediárias não foram observadas, indicando a formação de uma única fase cristalina.

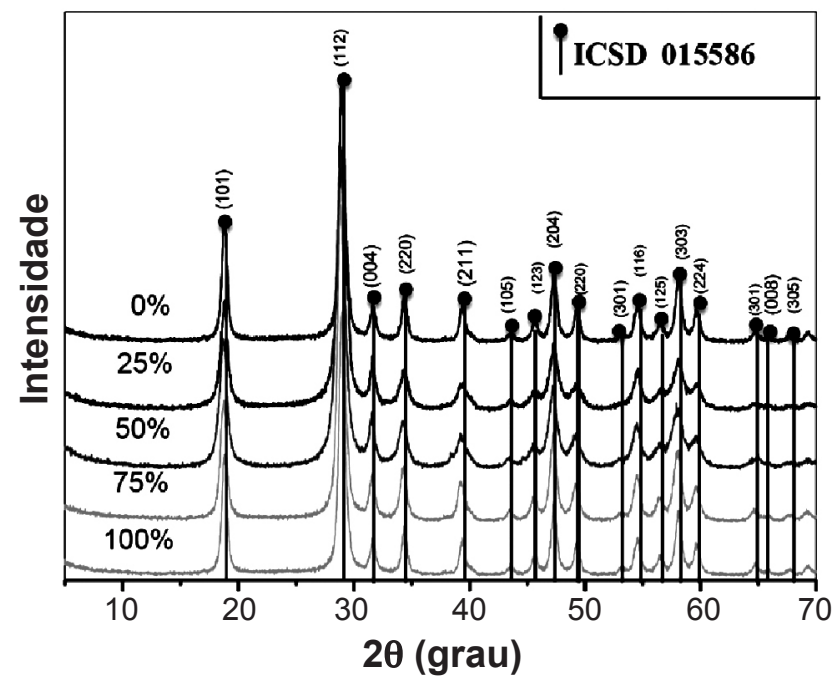

Figura 1: DRX dos $\mathrm{CaWO}_{4}$ sintetizados a temperatura ambiente. [Figure 1: XRD of $\mathrm{CaWO}_{4}$ synthesized room temperature.]
Com o programa X'Pert HighScore Plus (Versão 2.0.1 Windows) foi possível fazer a identificação dos picos com base nos arquivos ICSD. Todos os picos foram indexados com a estrutura do tipo scheelita tetragonal de grupo espacial I41/a de acordo com o arquivo ICSD 015586 [16]. Os dados referentes aos parâmetros de rede experimentais, bem como o volume da célula unitária, com o programa DBWSTool (Versão 2.3 Windows), foram calculados por meio do refinamento de Rietveld. Os valores obtidos em função das variações dos solventes são apresentados na Tabela I [17].

O método de Rietveld, de refinamento de estruturas cristalinas, consiste no ajuste dos dados obtidos pela difração de raios $\mathrm{X}$ e os dados são calculados por meio de um modelo fenomenológico, pelo método dos mínimos quadrados. O principal objetivo do método de Rietveld é refinar os valores dos parâmetros estruturais da amostra em pó com os resultados da difração, bem como obter os parâmetros que caracterizam a forma dos perfis dos picos de difração [18]. O ruído de fundo utilizando uma função polinomial, os parâmetros da temperatura isotrópica por átomo, os dados fracionários atômicos para o átomo de $\mathrm{O}$, os parâmetros de rede $(a, b, c)$, parâmetros do perfil de meia largura (u, v, w), ocupação dos sítios atômicos (Wyckoff) e os parâmetros de refinamento global (coeficientes polinomiais) foram ajustados. Os parâmetros a, b, c e o volume da célula unitárias em função das distintas quantidades de solventes são apresentados na Tabela I. A convergência foi acompanhada por alguns índices calculados ao final de cada ciclo de refinamento e que forneceram um subsídio necessário sobre a estrutura. Esses índices são o Rponderado $\left(\mathrm{R}_{\mathrm{wP}}\right)$, "goodness of fit" (S) e $R_{B R A G G}[19]$ ]. O indicador numérico $R w p$ é estatisticamente o mais significativo, pois o numerador é o resíduo a ser minimizado [20]. Os resultados obtidos de refinamentos estruturais para serem aceitáveis devem seguir os seguintes critérios: (a) $R w p<10 \%$ - fase média complexa (tetragonal,

Tabela I - Dados comparativos dos parâmetros de rede e volume da célula unitária para os cristais de $\mathrm{CaWO}_{4}$ sintetizados obtidos neste trabalho e outros citados na literatura para este material sintetizado por diferentes metodologias.

[Table I - Comparative data of lattice parameters and unit cell volume for the synthesized CaWO $\mathrm{Wrystals}_{4}$ obtained in this study and others cited in the literature for this material synthesized by different methods.]

\begin{tabular}{|c|c|c|c|c|c|c|}
\hline \multirow{2}{*}{$\begin{array}{ll} & \text { Método } \\
\text { SL } & \end{array}$} & \multirow{2}{*}{$\begin{array}{c}\begin{array}{c}\mathrm{T} \\
\left({ }^{\circ} \mathrm{C}\right)\end{array} \\
80\end{array}$} & \multirow{2}{*}{$\begin{array}{c}\mathrm{t} \\
(\mathrm{h}) \\
1\end{array}$} & \multicolumn{2}{|c|}{$\begin{array}{c}\text { Parâmetros de rede }(\AA) \\
a=b c\end{array}$} & \multirow{2}{*}{$\begin{array}{c}\mathrm{V}\left(\AA^{3}\right) \\
------\end{array}$} & \multirow{2}{*}{$\frac{\text { Ref. }}{[17]}$} \\
\hline & & & 5,2563 & 11,4007 & & \\
\hline MEHT & 180 & 12 & 5,2453 & 11,3715 & ------ & [4] \\
\hline MS & 950 & 10 & 5,2360 & 11,3580 & 311,39 & [5] \\
\hline $\mathrm{MCP}_{(x=}$ & [њ] & 0,5 & 5,2438 & 11,3679 & 312,59 & [o] \\
\hline $\operatorname{MCP}_{(x=0,25)}^{(x=0)}$ & [क] & 0,5 & 5,2440 & 11,3567 & 312,31 & [o] \\
\hline $\operatorname{MCP}_{(x=0,50)}^{(x-2,5)}$ & [\%] & 0,5 & 5,2458 & 11,3601 & 312,61 & [o] \\
\hline $\operatorname{MCP}_{(x=0,75)}^{(x=0,0)}$ & [њ] & 0,5 & 5,2390 & 11,3551 & 312,59 & [o] \\
\hline $\operatorname{MCP}_{(x=l)}^{(x=0, /)}$ & [م] & 0,5 & 5,2496 & 11,3643 & 313,18 & [o] \\
\hline $\operatorname{ICSD}^{(x-1)}$ & $\mathrm{N}^{\mathrm{o}} .015586$ & & & & & [-] \\
\hline
\end{tabular}

* Mistura contendo um total de 100 gramas de diferentes proporções em massa de solventes $\left(x \mathrm{H}_{2} \mathrm{O}: 1-x \mathrm{C}_{2} \mathrm{H}_{6} \mathrm{O}_{2}\right)$, ondex $=1 ; 0,75 ; 0,50 ; 0,25$ e $0 ; \mathrm{T}=$ temperatura; $t=$ tempo; [ Temperatura ambiente, a, b, $c=$ Parâmetro de rede, $V=$ Volume da Célula Unitária; Anglos $\alpha=\beta=\gamma=90^{\circ} ; S L=$ sonoquímica leve; MEHT = microemulsão hidrotermal; $M S=$ microondas-solvotermal, $M C P=$ método da coprecipitação, $[\mathbf{\square}]=$ ICSDe [o] = Este trabalho. 

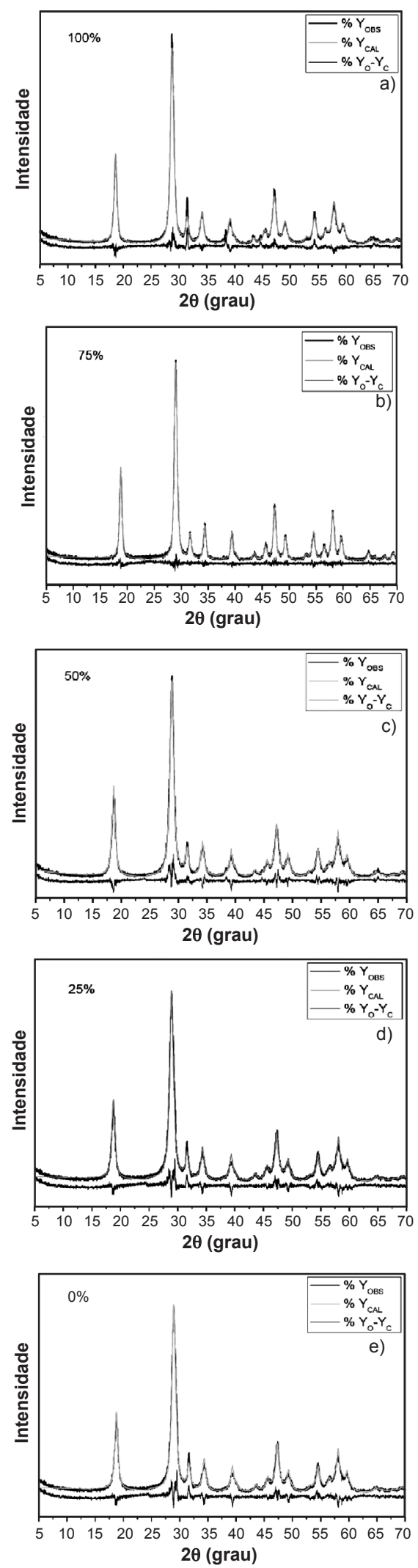

Figura 2: Difratogramas de raios $\mathrm{X}$ provenientes do refinamento de Rietveld dos óxidos sintetizados à temperatura ambiente, contendo as respectivas proporções mássicas do solvente etileno glicol.

[Figure 2: X-ray diffraction patterns from the Rietveld refinement of the oxides synthesized at room temperature containing the respective mass ratios of solvent ethylene glycol.] ortorrômbica, romboédrica e hexagonal), (b) $R w p<15 \%$ alta fase complexa (monoclínico e triclínico), (c) $R w p<8 \%$ - estrutura cúbica (simetria elevada com poucos picos de difração). Além disso, os baixos valores de $S(<2)$ são indicativos de uma boa precisão para os resultados obtidos de refinamento [21].

$\mathrm{R}_{\mathrm{EXP}}$ é o valor estatisticamente esperado para o $\mathrm{R}_{\mathrm{WP}}$ e o $\mathrm{S}\left(\mathrm{S}=\mathrm{R}_{\mathrm{WP}} / \mathrm{R}_{\mathrm{EXP}}\right)$ apresentou-se para todos os refinamentos entre 1,45 a 1,88 . Todos esses índices fornecem subsídios necessários para julgar a qualidade do refinamento. Para avaliar a qualidade do modelo estrutural refinada, devese calcular e verificar os valores dos parâmetros $\mathrm{R}_{\mathrm{BRAGG}}$ ou $R_{B}$, que é descrito como uma função das intensidades integradas dos picos ou um parâmetro isotrópico [18]. Como a intensidade integrada está relacionada com a estrutura cristalina (tipos de átomos, posições e deslocamentos atômicos), esse índice também deve ser considerado ao se avaliar a qualidade do modelo refinado da estrutura cristalina $[18,20]$. Desde que o parâmetro isotrópico está associado ao grau de ordem-desordem da estrutura, um maior valor do $R_{B}$ sugere um maior grau de desordem estrutural. Em geral, para um bom refinamento os valores de $\mathrm{R}_{\mathrm{BRAGG}}$, variam de $5 \%$ [19]. Porém, pode-se observar na Tabela II que alguns desses valores não estão dentro da margem de aceitação. Segundo Toby a integração usada para os índices $\mathrm{R}_{\mathrm{BRAGG}}$ começa a falhar quando os picos têm caudas longas ou têm assimetria não modelada significativa porque as partes do pico não estão incluídas no cálculo de intensidade. Na prática, isto não é um grande problema, pois os valores de $\mathrm{R}_{\mathrm{BRAGG}}$ não tem validade estatística [22]. As posições de Wyckoff para o átomo de $\mathrm{Ca}$ foram $4 b$, para o átomo de $\mathrm{W}$ foram $4 a$ e para o átomo de $\mathrm{O}$ foram $16 f$. Para os átomos de $\mathrm{Ca}$ os valores do parâmetro $x$ foram iguais à zero, os parâmetros $y$ foram iguais a 0,2500 ; os parâmetros $z$ foram iguais a 0,6250 ; os valores dos ângulos $(\alpha, \beta, \gamma)$ para a célula unitária foram iguais a $90^{\circ}(\alpha=\beta=\gamma)$. Para os átomos de $\mathrm{W}$, os valores dos parâmetros $x$ foram iguais à zero, os parâmetros $y$ foram iguais a 0,2500 e os parâmetros $z$ foram iguais a 0,1250 . Esses parâmetros variaram somente para os átomos de O. A Tabela II mostra os parâmetros obtidos no refinamento.

Com o programa Diamond Crystal and Molecular Structure Visualization (Versão 3.2e Windows) [23], juntamente com os parâmetros de rede, posições atômicas obtidas por meio do refinamento de Rietveld (Tabelas I e II) e alguns ajustes no software como, por exemplo, levandose em consideração o grupo espacial ocupado (I41/a) e o seu grupo de simetria $\left(\mathrm{C}_{4 \mathrm{~h}}^{6}\right)$ [24], foi possível fazer a representação da célula unitária no $\mathrm{CaWO}_{4}$ sintetizada e também as informações dos ângulos $(\alpha$ e $\beta$ ),como pode-se observar na Fig. 3.

A Fig. 3 é a representação da célula unitária do óxido sintetizado. Na célula unitária, os átomos de tungstato são coordenados a quatro oxigênios, formando os tetraédricos do tipo $\left[\mathrm{WO}_{4}\right]$. Além disso, com as informações dos ângulos $(\alpha$ e $\beta$ ) fornecidas pelo software, ou seja, ângulos de ligação entre $\mathrm{W}-\mathrm{O}$ e $\mathrm{O}-\mathrm{O}$, respectivamente foi possível perceber que os clusters $\left[\mathrm{WO}_{4}\right]$ são levemente distorcidos dentro 
Tabela II - Parâmetros (x, y, z) para os átomos de oxigênio, $\mathrm{R}_{\mathrm{WP}}, \mathrm{R}_{\mathrm{EXP}}, \mathrm{R}_{\mathrm{B}} \mathrm{e} \mathrm{S}$ obtidos no refinamento de Rietveld. [Table II - Parameters $(x, y, z)$ to the oxygen atoms, $R_{W P} R_{E X P}$ and $S$ obtained in the Rietveld refinement.]

\begin{tabular}{cccccccc}
\hline Proporções do Solvente $^{*}$ & $\mathrm{x}$ & $\mathrm{y}$ & $\mathrm{z}$ & $\mathrm{R}_{\mathrm{WP}}(\%)$ & $\mathrm{R}_{\mathrm{EXP}}(\%)$ & $\mathrm{R}_{\mathrm{B}}$ & $\mathrm{S}$ \\
\hline 1 & 0,1494 & $-0,0051$ & 0,2080 & 12,34 & 8,52 & 2,91 & 1,45 \\
0,75 & 0,1548 & $-0,0191$ & 0,2093 & 11,13 & 7,72 & 4,80 & 1,44 \\
0,5 & 0,1453 & 0,0027 & 0,2038 & 13,44 & 7,16 & 9,44 & 1,88 \\
0,25 & 0,1596 & $-0,0191$ & 0,2071 & 14,17 & 7,41 & 3,03 & 1,41 \\
0 & 0,1544 & $-0,0105$ & 0,2061 & 13,25 & 7,56 & 5,81 & 1,75 \\
\hline
\end{tabular}

${ }^{*}$ Mistura contendo um total de 100 gramas de diferentes proporções em massa de solventes $\left(\mathrm{xH}_{2} \mathrm{O}: 1-x \mathrm{C}_{2} \mathrm{H}_{6} \mathrm{O}_{2}\right)$, onde $x=1 ; 0,75 ; 0,50 ; 0,25$ e 0, ou seja, variação de $100 \mathrm{a} 0 \%$ de $\mathrm{C}_{2} \mathrm{H}_{6} \mathrm{O}_{2}$.

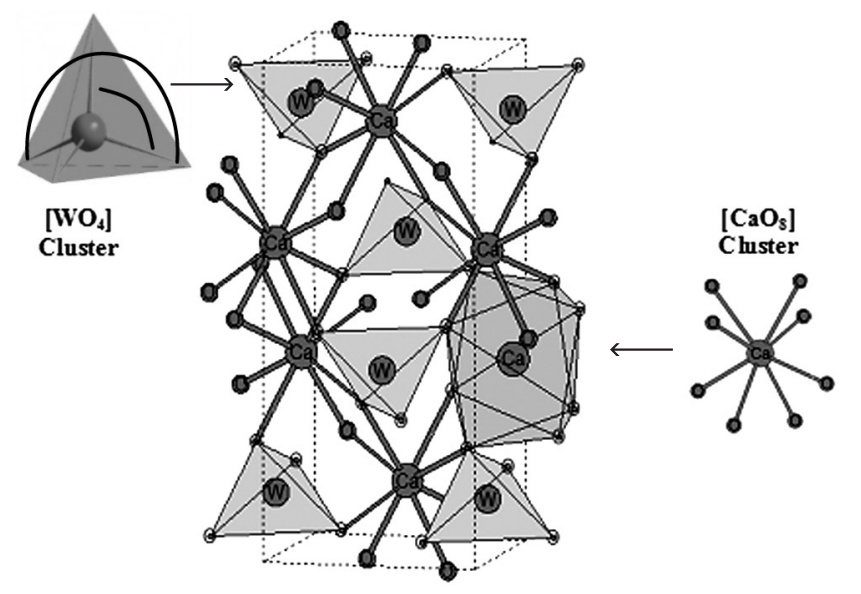

Figura 3: Representação esquemática das células unitárias correspondente ao $\mathrm{CaWO}_{4}$ sintetizados.

[Figure 3: Schematic representation of the unit cells corresponding to CaWO4 synthesized.]

Tabela III - Informações dos ângulos $\alpha$ e $\beta$ para os clusters do tipo $\mathrm{WO}_{4}$.

[Table III - Information of the angles $\alpha$ and $\beta$ for the type $\mathrm{WO}_{4}$ clusters.]

\begin{tabular}{cccc}
\hline $\begin{array}{c}\text { Proporções do } \\
\text { Solvente }\end{array}$ & $\alpha(\mathrm{W}-\mathrm{O})$ & $\beta(\mathrm{O}-\mathrm{O})$ & Ref. \\
\hline------ & $107,88^{\circ}$ & $112,69^{\circ}$ & {$[26]$} \\
----- & $107,25^{\circ}$ & $114,01^{\circ}$ & {$[20]$} \\
1 & $105,690^{\circ}$ & $117,330^{\circ}$ & {$[\square]$} \\
0,75 & $104,887^{\circ}$ & $119,090^{\circ}$ & {$[\square]$} \\
0,5 & $105,155^{\circ}$ & $118,499^{\circ}$ & {$[\square]$} \\
0,25 & $104,147^{\circ}$ & $120,742^{\circ}$ & {$[\square]$} \\
0 & $104,570^{\circ}$ & $119,793^{\circ}$ & {$[\square]$} \\
\hline
\end{tabular}

"Mistura contendo um total de 100 gramas de diferentes proporções em massa de solventes $\left(x_{2} \mathrm{O}: 1-x \mathrm{C}_{2} \mathrm{H}_{6} \mathrm{O}_{2}\right)$,

onde $x=1 ; 0,75 ; 0,50 ; 0,25$ e 0 , ou seja, variação de 100 a $0 \%$ de $\mathrm{C}_{2} \mathrm{H}_{6} \mathrm{O}_{2}$. $[\square]=$ Neste trabalho.

da rede dos óxidos não tratados termicamente (Tabela III). Pode-se dizer que as condições reacionais como, por exemplo, a variação das proporções adequadas do solvente utilizado podem ter afetado diretamente essas pequenas distorções dos clusters dos óxidos sintetizados. Os cristais do tipo scheelita apresentam sua célula primitiva formada pelos grupos iônicos $\left(\mathrm{WO}_{4}^{2-}\right)$ e o $\left(\mathrm{Ca}^{2+}\right)$. A estrutura cristalina apresenta caráter iônico para os cátions $\left(\mathrm{Ca}^{2+}\right)$ e carácter covalente para os grupos $\left(\mathrm{WO}_{4}^{2-}\right)[25]$.

Na Fig. 3 os átomos de tungstato estão coordenados a quatro oxigênios, formando os aglomerados tetraédricos de $\mathrm{WO}_{4}$ que possuem 4 vértices, 4 faces e arestas 6. Os átomos de cálcio, dentro da scheelita, são coordenados a oito átomos de oxigênio, ou seja, cluster do tipo $\mathrm{CaO}_{8}$, adotando a forma de um poliedro não uniformes convexos da forma biesfenoide como que possuem 8 vértices, 12 faces e 18 arestas, com coordenação deltraedro e ponto-grupo simetria $\mathrm{D}_{2 \mathrm{~d}}$ [27]. Estes clusters diedros também apresentam distorções angulares, no entanto, são mais complexas do que o detectado para aqueles os clusters de $\mathrm{WO}_{4}$ [20].

A espectroscopia Raman e a FT-IV foram utilizadas para identificar as unidades estruturais dos pós com base na freqüência vibracionais das moléculas. Os cálculos da teoria de grupo sugerem a presença de 26 diferentes vibrações para cristais de $\mathrm{CaWO}_{4}$, que podem ser representados pela equação $\mathrm{A}[28,29]$ :

$$
\Gamma_{\text {(Raman }+ \text { Infravermelho) }}=3 A_{g}+5 A_{u}+5 B_{g}+3 B_{u}+5 E_{g}+5 E_{u},
$$

na qual os símbolos $A_{g}$, $B_{g}$ e $E_{g}$ representam modos vibracionais ativos no Raman, sendo os modos A e B nãodegenerados, enquanto que os modos $\mathrm{E}$ são duplamente degenerados. Os subscritos " $g$ e $u$ " indicam operações de simetria relacionadas à inversão do centro-simétrico do cristal de $\mathrm{CaWO}_{4}$, sendo os modos com subscrito " $u$ " observados apenas no infravermelho. Os modos $A_{u}$ e $E_{u}$ correspondem ao zero de frequência de modos acústicos, ao passo que os modos $B_{u}$ são modos ópticos. Entretanto, os modos $A_{g}, B_{g}$ e $E_{g}$ estão relacionados a movimentos de vibração dentro dos cristais de $\mathrm{CaWO}_{4}$. Portanto, 13 modos ativos são esperados no espectro de espalhamento Raman para cristais de $\mathrm{CaWO}_{4}$, representados pela Equação (B) [28-30].

$$
\Gamma_{\text {(Raman) }}=3 A_{g}+5 B_{g}+5 E_{g},
$$

As unidades formulares de $\mathrm{CaWO}_{4}$ são caracterizadas por grupos moleculares iônicos $\left[\mathrm{WO}_{4}^{2-}\right]$ e cátions $\mathrm{Ca}^{2+}$. Os grupos moleculares, ou seja, os "clusters" [ $\left[\mathrm{WO}_{4}\right]$, 
com fortes ligações covalentes são peculiares devido ao fraco acoplamento entre os grupos moleculares [ $\left.\mathrm{WO}_{4}\right]$ e os cátions $\mathrm{Ca}^{2+}$, os modos vibracionais visualizados no espectro Raman de molibdatos podem ser classificados em dois grupos: modos internos e modos externos [31, 32]. No espectro vibracional na região do infravermelho, dois modos acústicos $\left(1 \mathrm{~A}_{u}\right.$ e $\left.1 \mathrm{E}_{u}\right)$ são inativos enquanto que os modos ópticos $\mathrm{B}_{u}$ são proibidos. Por esta razão, apenas 8 vibrações ativas remanescentes, representadas pela Equação (C), são esperadas no espectro infravermelho [33, 34]:

$$
\Gamma_{\text {(Infravermelho) }}=4 A_{u}+4 E_{u},
$$

A Fig. 4 mostra os espectros Raman na região de 75 a $1100 \mathrm{~cm}^{-1}$ para o óxido de $\mathrm{CaWO}_{4}$ preparado pelo método de co-precipitação.

Dez diferentes modos de vibração $\left(2 \mathrm{~A}_{\mathrm{g}}, 5 \mathrm{~B}_{\mathrm{g}}\right.$ e $\left.3 \mathrm{E}_{\mathrm{g}}\right)$ foram detectados nos espectros Raman (Fig. 4), a Tabela IV mostra

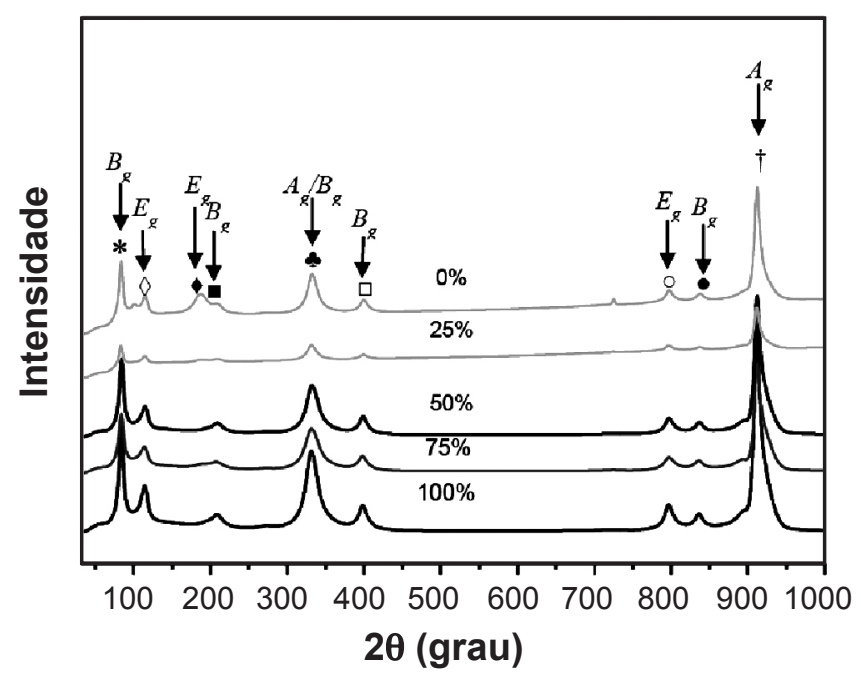

Figura 4: Espectro Raman na região de 75 a $1000 \mathrm{~cm}^{-1}$ para os nanocristais de $\mathrm{CaWO}_{4}$ sintetizado a temperatura ambiente com a proporção mássica do etileno glicol variado entre 0 a $100 \%$.

[Figure 4: Raman spectrum in the region 75 to $1000 \mathrm{~cm}^{-1}$ for $\mathrm{CaWO}_{4}$ nanocrystals synthesized at room temperature with the weight ratio of the ethylene glycol varied from 0 to $100 \%$.] os valores obtidos para cada modo vibracional.

Os resultados expressos na Tabela IV exploram os modos ativos no espectro de Raman obtido neste trabalho comparado ao já reportados com a literatura. Três modos vibracionais $\left(1 \mathrm{~A}_{\mathrm{u}}\right.$ e $\left.2 \mathrm{E}_{\mathrm{g}}\right)$ não foram detectados devido a sua baixa intensidade. Pequenos deslocamentos notados nos modos Raman podem estar correlacionados a diferentes fatores, como: distribuição granulométrica dos cristais, distorções e força de interação entre "clusters" $\left[\mathrm{WO}_{4}\right]$ e/ou grau de ordem, distorções nas ligações $(\mathrm{O}-\mathrm{W}-\mathrm{O}) /(\mathrm{O}-\mathrm{Ca}-\mathrm{O})$, as forças de interação envolvendo os clusters $\left[\mathrm{WO}_{4}\right]-\left[\mathrm{CaO}_{8}\right]$ - $\left[\mathrm{WO}_{4}\right]$ e/ou e desordem estrutural no retículo cristalino [20]. Os modos de vibração de maior energia referem-se aos modos simétricos e assimétricos de estiramento entre o $\mathrm{W}-\mathrm{O}$ e as energias menores referem-se ao modo de flexão (torção) entre o $\mathrm{W}-\mathrm{O}$ e entre $\mathrm{Ca}-\mathrm{O}$.

A Fig. 5 mostra os espectros FT-IV na região de 400 a $1300 \mathrm{~cm}^{-1}$ para o óxido de $\mathrm{CaWO}_{4}$ preparado pelo método de co-precipitação sintetizados a temperatura ambiente. Os tungstatos com estrutura tetragonal apresentam modos ativos no infravermelho, relacionados a vibrações de estiramento e flexões antissimétricos de suas ligações. Os tungstatos com tipo estrutural scheelita possuem oito modos vibracionais correspondentes a alongamento e/ou flexão quando é submetido às radiações do tipo infravermelho [20].

Foram identificados três modos de vibração nos espectros: um modo do tipo $\mathrm{E}_{\mathrm{u}}$, um modo do tipo $\mathrm{A}_{\mathrm{u}}$ e um do tipo $\left[\mathrm{E}_{\mathrm{u}}+\mathrm{A}_{\mathrm{u}}\right.$ ]. Os cristais de $\mathrm{CaWO}_{4}$ exibiram uma sobreposição de duas bandas de absorção muito intensas, do tipo $v_{3}\left(\mathrm{~A}_{u}\right)$ e $v_{3}\left(\mathrm{E}_{\mathrm{u}}\right)$, nas regiões de $796 \mathrm{~cm}^{-1}$ a $806 \mathrm{~cm}^{-1}$ (Fig. 5) que foram atribuídos as vibrações antissimétricas do tipo $(\rightarrow \mathrm{O}-\mathrm{W}-\mathrm{O} \rightarrow)$, ou seja,vibração de estiramento antissimétrico dos "clusters" tetraédricos $\left[\mathrm{WO}_{4}\right][20,37]$.Os modos vibracionais $\mathrm{v}_{4}\left(\mathrm{~A}_{\mathrm{u}}\right.$ $\left.+\mathrm{E}_{\mathrm{u}}\right)$ situados entre $437 \mathrm{~cm}^{-1} \mathrm{a} 441 \mathrm{~cm}^{-1}$ que correspondem a vibrações simétricas do tipo alongamento $(\leftarrow \mathrm{O}-\mathrm{W}-\mathrm{O} \rightarrow$ ) dentro dos "clusters" tetraédricos $\left[\mathrm{WO}_{4}\right]$ [25]. A Tabela $\mathrm{V}$ mostra os valores obtidos e os compara com alguns já anteriormente reportados na literatura.

Pode-se afirmar que há extrema consonância entre os resultados deste trabalho e os outros já publicados, ou seja,

Tabela IV - Freqüência vibracionais dos modos ativos Raman para os pós de $\mathrm{CaWO}_{4}$ sintetizados.

[Table IV - Frequency vibrational modes of Raman active for $\mathrm{CaWO}_{4}$ powders synthesized.]

\begin{tabular}{|c|c|c|c|c|c|c|c|c|c|c|c|c|c|}
\hline Método & $\begin{array}{c}\mathrm{T} \\
\left({ }^{\circ} \mathrm{C}\right)\end{array}$ & $\begin{array}{c}\mathrm{t} \\
\text { (h) }\end{array}$ & $B_{g}$ & $\begin{array}{c}E_{g} \\
\diamond^{\prime}\end{array}$ & $E_{g}$ & $B_{g}$ & $\begin{array}{l}A_{g} \\
\stackrel{5}{*}\end{array}$ & $\begin{array}{l}B_{g} \\
\omega^{2}\end{array}$ & $\begin{array}{c}B_{g} \\
\square\end{array}$ & $\begin{array}{c}E_{g} \\
0\end{array}$ & $B_{g}$ & $\stackrel{A_{g}}{\grave{\dagger}}$ & Ref. \\
\hline $\mathrm{MC}$ & ---- & ---- & 84 & 118 & 195 & 212 & 333 & 333 & 401 & 797 & 838 & 911 & [35] \\
\hline MCBD & 80 & 13 & 82 & ---- & ---- & 208 & 330 & 330 & ---- & 797 & 835 & 911 & [36] \\
\hline $\mathrm{MCP}_{(x=0)}$ & 25 & 0,5 & 84 & 114,5 & 188 & 209 & 332 & 332 & 399,5 & 797 & 838,5 & 913 & {$[\square]$} \\
\hline $\mathrm{MCP}_{(x=0,25)}$ & 25 & 0,5 & 82 & 114,5 & 186 & 205,5 & 330,5 & 330,5 & 401,5 & 799 & 838,5 & 911 & [ם] \\
\hline $\mathrm{MCP}_{(x=0,50)}$ & 25 & 0,5 & 84 & 115 & 189,5 & 207,5 & 332,5 & 332,5 & 399,5 & 797 & 836,5 & 911 & {$[\square]$} \\
\hline $\operatorname{MCP}_{(x=0,75)}^{(x-0,0)}$ & 25 & 0,5 & 84 & 115 & 189,5 & 207,5 & 332,5 & 332,5 & 397,5 & 797 & 836,5 & 913 & [ם] \\
\hline $\mathrm{MCP}_{(x=1)}$ & 25 & 0,5 & 84 & 115 & 186 & 207,5 & 332,5 & 332,5 & 397,5 & 797 & 836,5 & 915 & {$[\square]$} \\
\hline
\end{tabular}

Mistura contendo um total de 100 gramas de diferentes proporções em massa de solventes $\left(\mathrm{xH}_{2} \mathrm{O}: 1-x \mathrm{C}_{2} \mathrm{H}_{6} \mathrm{O}_{2}\right)$, onde $x=1 ; 0,75 ; 0,50 ; 0,25$ e 0. MH $=$ método hidrotermal; $M C=$ Método computacional, $M C B D=$ Método do banho de posição química, SN = suspensão nanocoloidal e [ $\square$ ] = Este trabalho. 


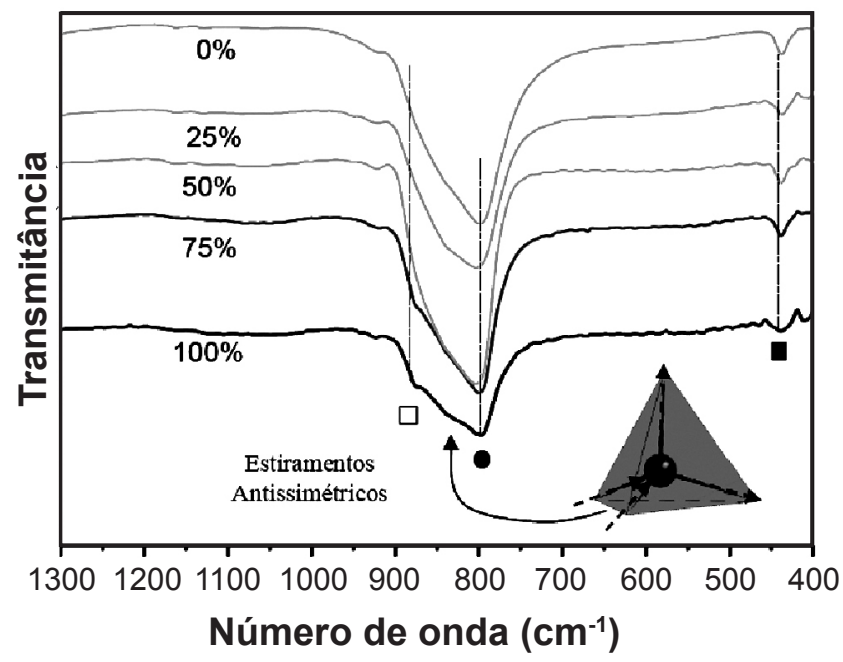

Figura 5: Espectro FT-IV na região de 400 a $1300 \mathrm{~cm}^{-1}$ para os nanocristais de $\mathrm{CaWO}_{4}$ sintetizado a temperatura ambiente com a proporção mássica do etileno glicol variando entre 0 a $100 \%$.

[Figure 5: FT-IR spectrum in the region 400 to $1300 \mathrm{~cm}^{-1}$ for CaWO4 nanocrystals synthesized at room temperature with the weight ratio of ethylene glycol ranging from 0 to $100 \%$.]

Tabela V - Freqüências vibracionais dos modos ativos IV para os pós de $\mathrm{CaWO}_{4}$ sintetizados.

[Table $V$ - Vibrational frequency modes to the active $I V$ $\mathrm{CaWO}_{4}$ synthesized powders.]

\begin{tabular}{lcccccc}
\hline Método & $\begin{array}{c}\mathrm{T} \\
\left({ }^{\circ} \mathrm{C}\right)\end{array}$ & $\begin{array}{c}\mathrm{t} \\
(\mathrm{h})\end{array}$ & $\begin{array}{c}A_{u}+E_{u} \\
\left(\mathrm{~cm}^{-1}\right)\end{array}$ & $\begin{array}{c}E_{u} \\
\left(\mathrm{~cm}^{-1}\right) \\
\bullet\end{array}$ & $\begin{array}{c}A_{u} \\
\left(\mathrm{~cm}^{-1}\right)\end{array}$ & Ref. \\
\hline $\mathrm{CZ}$ & 1100 & 24 & 366 & 796 & ---- & {$[38]$} \\
$\mathrm{MH}$ & 120 & 12 & 442 & 799 & 839 & {$[1]$} \\
$\mathrm{CP}$ & 25 & 24 & 461 & ---- & 966 & {$[39]$} \\
$\mathrm{MEHT}$ & 180 & 12 & 438 & 788 & ---- & {$[4]$} \\
$\mathrm{MCP}_{(x=0)}$ & {$[\bullet]$} & 0,5 & 436 & 796 & 877 & {$[\square]$} \\
$\mathrm{MCP}_{(x=0,25)}$ & {$[\bullet]$} & 0,5 & 437 & 801 & 880 & {$[\square]$} \\
$\mathrm{MCP}_{(x=0,50)}$ & {$[\bullet]$} & 0,5 & 437 & 801 & 879 & {$[\square]$} \\
$\operatorname{MCP}_{(x=0,75)}$ & {$[\bullet]$} & 0,5 & 437 & 801 & 846 & {$[\square]$} \\
$\operatorname{MCP}_{(x=1)}$ & {$[\bullet]$} & 0,5 & 437 & 796 & 862 & {$[\square]$} \\
\hline
\end{tabular}

Mistura contendo um total de 100 gramas de diferentes proporções em massa de solventes $\left(x \mathrm{H}_{2} \mathrm{O}: 1-x \mathrm{C}_{2} \mathrm{H}_{6} \mathrm{O}_{2}\right)$, onde $x=1 ; 0,75 ; 0,50 ; 0,25$ e 0. $T=$ temperatura; $t=$ tempo; $[\mathbf{0}]=$ Temperatura ambiente, $C Z=$ Czochralski; $C P=$ Co-precipitação; $M S=$ solvotermal; $M H=$ hidrotermal; $M S H=$ solvotermal-hidrotermal $e[\square]=$ Este trabalho.

a eficiência do método empregado, visto que aplicando condições experimentais mais brandas, foi possível observar alguns modos similares aos que já foram anteriormente reportados pela literatura quando se foi empregado condições reacionais um pouco mais enérgicas.

A Fig. 6 mostra os espectros de absorção UV-Vis dos nanocristais de $\mathrm{CaWO}_{4}$ preparados em diferentes proporções de solventes $\left(\mathrm{H}_{2} \mathrm{O} / \mathrm{C}_{2} \mathrm{H}_{6} \mathrm{O}_{2}\right)$. Os valores dos "band gap" ópticos obtidos em função das diferentes proporções de
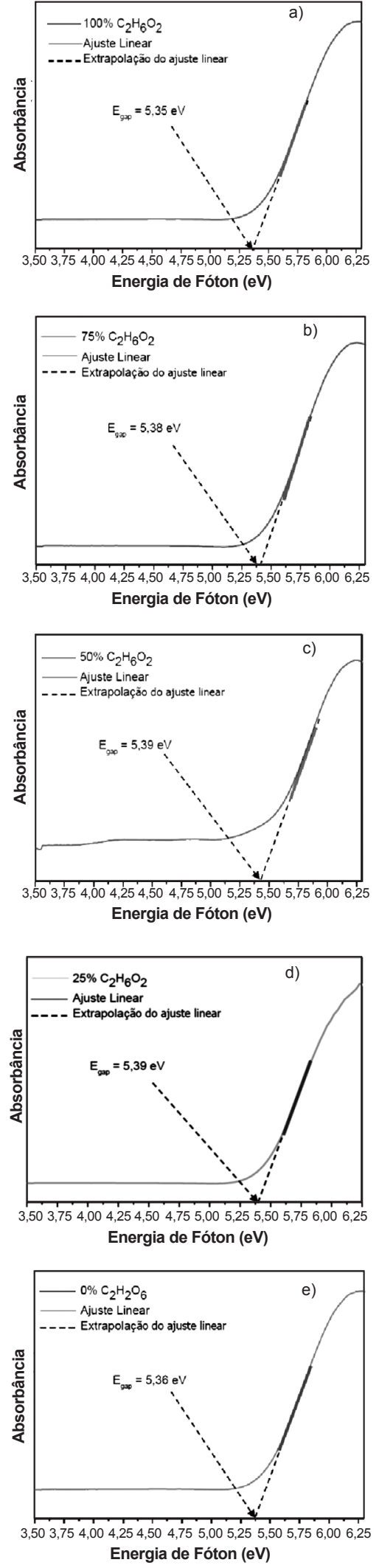

Figura 6: Espectro de absorção na região do UV-vis dos nanocristais $\mathrm{CaWO}_{4}$ sintetizados a temperatura ambiente utilizando com a proporção mássica do etileno glicol variando entre 0 a $100 \%$.

[Figure 6: Absorption spectrum in the UV-vis of $\mathrm{CaWO}_{4}$ nanocrystals synthesized at room temperature using the same proportion by mass of ethylene glycol ranging from 0 to $100 \%$.] 
solventes são resumidos na Tabela VI.

A energia de "band gap" óptico $\left(\mathrm{E}_{\text {gap }}\right)$ foi estimada pelo método proposto por Wood e Tauc [40]. De acordo com estes autores, a $\mathrm{E}_{\text {gap }}$ está associada com a absorbância e a energia do fóton. Em uma estrutura de banda parabólica, o espaçamento entre as bandas óptico e coeficiente de absorção de óxidos de semicondutores pode ser calculada pela seguinte equação:

$$
\alpha \mathrm{h} v \propto \mathrm{C}_{1}\left(\mathrm{~h} v-\mathrm{E}_{\mathrm{gap}}\right)^{\mathrm{n}}
$$

na qual $\alpha$ é o coeficiente de absorção linear do material, hv é a energia do fóton, $\mathrm{C}_{1}$ é a constate de proporcionalidade, $\mathrm{E}_{\text {gap }}$ é a diferença da "band gap" óptica e $n$ é uma constante associada à diferentes tipo de transições eletrônicas $(n=1 / 2,23 / 2$ ou 3 para transições diretas permitidas, indiretas permitidas, diretas proibidas e indiretas proibidas, respectivamente).

Os molibdatos e tungstatos têm um típico processo de absorção óptica caracterizado por transições eletrônicas diretas, que ocorrem de estados de energia máximos localizados próximos ou na banda de valência para mínimos estados de energia localizados abaixo ou na banda de condução, nas mesmas regiões na zona de Brillouin [41, 42].

Os valores de $\mathrm{E}_{\text {gap }}$ dos nano e mesocristais de $\mathrm{CaWO}_{4}$ foram calculados usando $n=1 / 2$ ou 0,5 na Equação $D$ e extrapolando a porção linear da curva ou cauda. Em geral, os materiais mais cristalinos apresentaram um maior grau de organização à média distância, quando comparados com materiais menos cristalinos. Assim, esses materiais apresentam elevado valor da energia "gap" óptica, devido apresentarem menos níveis intermediários de energia entre $\mathrm{BV}$ e $\mathrm{BC}$, resultando num maior intervalo de energia [43]. Neste caso, é necessário que esse sistema tenha um maior conteúdo energético para ocorrer às transições eletrônicas. Diferentes dos materiais

Tabela VI - Valores da $\mathrm{E}_{\text {gap }}$ obtida para os nanocristais de $\mathrm{CaWO}_{4}$ comparados com alguns valores reportados pela literatura.

[Table VI - Values obtained $E_{\text {gap }}$ for $\mathrm{CaWO}_{4}$ nanocrystals compared with some values reported in the literature.]

\begin{tabular}{ccccc}
\hline Método & $\begin{array}{c}\mathrm{T} \\
\left({ }^{\circ} \mathrm{C}\right)\end{array}$ & $\begin{array}{c}\mathrm{t} \\
(\mathrm{h})\end{array}$ & $\mathrm{E}_{\text {gap }}(\mathrm{eV})$ & Ref. \\
\hline $\mathrm{SNC}$ & ---- & 6 & 5,16 & {$[44]$} \\
$\mathrm{RES}$ & 800 & 5 & 4,50 & {$[45]$} \\
$\mathrm{PP}$ & 400 & 4 & 5,27 & {$[46]$} \\
$\mathrm{MCP}_{(x=0)}$ & 25 & 0,5 & 5,36 & {$[\square]$} \\
$\mathrm{MCP}_{(x=0,25)}$ & 25 & 0,5 & 5,39 & {$[\square]$} \\
$\mathrm{MCP}_{(x=0,50)}$ & 25 & 0,5 & 5,39 & {$[\square]$} \\
$\operatorname{MCP}_{(x=0,75)}$ & 25 & 0,5 & 5,38 & {$[\square]$} \\
$\operatorname{MCP}_{(x=1)}$ & 25 & 0,5 & 5,35 & {$[\square]$} \\
\hline
\end{tabular}

Mistura contendo um total de 100 gramas de diferentes proporções em massa de solventes $\left(x_{2} \mathrm{O}_{2}: 1-\mathrm{xC}_{2} \mathrm{H}_{6} \mathrm{O}_{2}\right), \quad$ onde $x=1 ; 0,75 ; 0,50 ; 0,25$ e 0. $T=$ temperatura; $t=$ tempo; $M S=$ solvotermal; $S N C=$ Suspensão nanocoloidal; $R E S=$ reação de estado sólido; $P P=$ percussores poliméricos; $M C P=$ método da coprecipitação e $[\square]=$ Este trabalho. mais desordenados, já que os mesmo apresentam um maior número de níveis intermediários, e com isso, o intervalo entre as bandas se torna menor e é necessária menor energia para ocorrer às transições eletrônicas [25].

Os valores contidos na Tabela IV estão bem próximos aos já reportados. Os valores da energia "band gap" óptica está associado com o grau de ordem e desordem estrutural dos materiais à média distância. Essa relação provocou pequenas diferenças nas densidades de defeito material, que acabaram por resultar em diferentes distribuições de níveis intermediários de energia entre a banda de valência (BV) e a banda de condução (BC) [47, 48]. Essas relações de ordem e desordem estrutural observadas entre os pós de $\mathrm{CaWO}_{4}$ processados a temperatura ambiente se devem às interações dos clusters de $\mathrm{Ca}-\mathrm{O}$ e W-O. Essas interações podem causar distorções nos ângulos e comprimentos das ligações, ou seja, em diferentes graus de defeitos, que resultaram em diferentes distribuições de níveis intermediários de energia entre a $\mathrm{BV}$ e BC $[25,47,48]$. As interações mais importantes, ou seja, as interações que influenciam significativamente na resposta do "gap" são as que ocorrem nos clusters de tungstato, já que as transições eletrônicas ocorrem dentro desse grupo tetraédrico [25, 43]. Essas relações de distorções nos ângulos e comprimento das ligações para o $\mathrm{WO}_{4}$ e $\mathrm{CaO}_{8}$, foram discutidas na seção na qual se discute sobre a representação das células unitárias do $\mathrm{CaWO}_{4}$ sintetizado.

\section{CONCLUSÕES}

A metodologia de síntese permitiu a obtenção do pós de $\mathrm{CaWO}_{4}$. Os espectros de DRX e Raman indicaram que os óxidos de $\mathrm{CaWO}_{4}$ são ordenados a médio e longo alcance com estrutura tetragonal tipo scheelita livre de fase secundária. $\mathrm{O}$ refinamento de Rietveld mostrou uma boa convergência entre os espectros de DRX obtidos experimentalmente com os descritos anteriormente na literatura. A simulação das células unitárias mostrou o grupo espacial I41/a e o grupo de simetria $\left(\mathrm{C}_{4 \mathrm{~h}}^{6}\right)$ que o óxido pertence como, também, os ângulos $\alpha$ e $\beta$ dos "clusters" $\left[\mathrm{WO}_{4}\right]$ dentro da rede cristalina. Esses valores estão bem próximos dos expostos pela literatura. Dos trezes modos ativos na espectroscopia Raman, dez diferentes modos de vibrações, sendo $2 \mathrm{~A}_{\mathrm{g}}, 5 \mathrm{~B}_{\mathrm{g}}$ e $3 \mathrm{E}_{\mathrm{g}}$, foram observados e somente três, do tipo $1 \mathrm{~A}_{\mathrm{u}} \mathrm{e} 2 \mathrm{E}_{\mathrm{g}}^{\mathrm{g}}$, não foram detectados devido a sua baixa intensidade. Os espectros FT-IV apresentaram três sinais: um modo do tipo $E_{u}$, um modo do tipo $A_{u}$ e um do tipo $\left[\mathrm{E}_{\mathrm{u}}+\mathrm{A}_{\mathrm{u}}\right]$ característico para estes materiais relacionados com vibrações assimétricas da ligação $\mathrm{W}-\mathrm{O}$. Os espectros de absorção UV-vis mostraram que os valores de "band gap" encontram-se próximos do relatados. A variação do solvente etileno glicol não influenciou nos parâmetros estruturais dos pós, porém a fotoluminescência, principal propriedade desse tipo de material, será estudada posteriormente.

\section{AGRADECIMENTOS}

Os autores agradecem ao CNPq, a CAPES e a FAPEPI. Agradecem também ao aluno de graduação em Química 
André Lima e Silva, pelo treinamento no método de refinamento de Rietveld.

\section{REFERÊNCIAS}

[1] Y. Su, G. Li, Y. Xue, L. Li. J. Phy. Chem. 111 (2007) 6684.

[2] F. R. C. Ciaco, F. M. Pontes, C. D. Pinheiro, E. R. Leite, R. S. Lazarro, J. A. Varela, C. A. Paskocimas, A. G. Souza, Cerâmica 50, 313 (2004) 43.

[3] Y. Su, L. Li, G. Li. Chem. Mater. 20 (2008) 6060.

[4] W. Wang, P. Yang, S. Gai,N. Niu, F. He, J. Lin, J. Nanopart. Res. 12 (2010) 2295.

[5] Z. Shan, Y. Wang, H. Ding, J. Mol. Catal. A: Chem. 302 (2009) 54.

[6] I. Trabelsi, M. Dammak, R. Maâlej, M. Kamoun, Physica B406 (2011) 315.

[7] B. A. Hernandez-Sanchez, T. J. Boyle, D. Harry, H. D. Pratt III, M. A. Rodriguez, L. N. Brewer, D. R. Dunphy, Chem. Mater. 20 (2008) 6643.

[8] L. Chen, Y. Gao, J. Zhu, Mater. Lett. 62 (2008) 3434.

[9] G. Lasse, B. C. Grabmeter, Luminescent Materials, Springer-Verlag, Berlin, Alemanha (1994) 232 p.

[10] B. S. Barros, A. C. de Lima, Z. R. da Silva, D. M. A. Melo, S. Alves-Jr. J. Phys. Chem. Solids 73 (2012) 635.

[11] A. Deoliveira, J. Ferreira, M. Silva, G. Braga, L. Soledade, M. Aldeiza, C. Paskocimas, S. Lima, E. Longo, A. G. de Souza, Dye Pigments 77 (2008) 210.

[12] J. C. Sczancoski, L. S. Cavalcante, M. R. Joya, J. W. M. Espinosa, P. S. Pizani, J. A. Varela, E. Longo, J. Coll. Interface Sci. 330 (2009) 227.

[13] C. Du, G. Yi, Y. Su, Z. Liu, J. Mater. Sci. 47 (2012) 6305.

[14] M. Kakihana, T. Okubo, M. Arima, Y. Nakamura, M. Yashima, M. Yoshimura,. J. Sol-gel Sci. Technol. 12 (1998) 95.

[15] V. S. Marques, L. S. Cavalcante, J. C. Sczancoski, A. F. P. Alcântara, M. O. Orlandi, E. Moraes, E. Longo, J. A. Varela, M. Siu Li, M. R. M. C. Santos, Cryst. Growth Design 10 (2010) 4752.

[16] A. Zalkin, D. H. Templeton, J. Chem. Phys. 40 (1964) 501.

[17] L. Bleicher, J. M. Sasaki, C. O. P. Santos, J. Appl. Cryst. 33 (2000) 1189.

[18] S. Lanfredi, M. A. L. Nobre, Quim. Nova 33 (2010) 1071.

[19] A. B. Campos, E. Longo, A. Z. Simões, J. A. Varela, V. M. Longo, A. T. Figueredo, F. de Vicente, S. A. C. Hernandes, Appl. Phys. Lett. 91 (2007) 051923-1.

[20] J. C. Araújo, L. Sena, I. N. Bastos, G. D. A. Santos, Quim. Nova 30 (2007) 1853.

[21] L. S. Cavalcante, V. M. Longo, J. C. Sczancoski, M. A. P. Almeida, A. A. Batista, J. A. Varela, M. O. Orlandi, E. Longo, M. Siu Li, Cryst. Eng. Comm. 14 (2012) 853.

[22] B. H. Toby, Powder Diffr. 21 (2006) 67.

[23] Diamond. Crystal and Molecular Structure
Visualization. Disponível em: <http://www.crystalimpact. com/ diamond/>. acesso em 21/08/2012.

[24] J. H. Ryu, J. W. Yoon, C. S. Lim, W. C. Oh, K. B. Shim, Ceram. Int. 31 (2005) 883.

[25] L. S. Cavalcante, J. C. Sczancoski, L. F. Lima Jr, J. W. M. Espinosa, P. S. Pizani, J. A. Varela, E. Longo, Cryst. Growth Des. 9 (2009) 1002.

[26] L. S. Cavalcante, J. C. Sczancoski, J. M. E. Matos, J. A. Varela, E. Longo, Mater. Sci. Eng. B 150 (2008) 18.

[27] Wikipédia, Deltahedro, disponível em: <http:// en.wikipedia.org/wiki/Deltahedron>, acesso em 21/08/2012. [28] S. P. S. Porto, J. F. Scott, Phys. Rev. 157 (1967) 716.

[29] D. Christofilos, G. A. Kourouklis, S. Ves, J. Phys. Chem. Solids 56 (1995) 1122.

[30] E. Sarantopoulou, C. Raptis, S. Ves, D. Christofilos, G. A. Kourouklis, J. Phys. - Condens. Matter. 14 (2002) 8925.

[31] T. T. Basiev, A. A. Sobol, P. G. Zverev, L. I. Ivleva, V. V. Osiko, Opt. Mater. 11 (2009) 307.

[32] T. T. Basiev, A. A. Sobol, Y. K. Voronko, P. G. Zverev, Opt. Mater. 15 (2000) 205.

[33] A. S. Barker Jr, Phys. Rev. 135 (1964) A742.

[34] A. Golubović, R. Gajić, Z. Dohćević-Mitrović, S. Nikolić, J. Alloys Compnd. 415 (2006) 16.

[35] L. Gracia, V. M. Longo, L. S. Cavalcante, A. Beltrán, W. Avansi, M. S. Li, V. R. Mastelaro, J. A. Valera, E. Longo, J. Andrés, J. Appl. Phys. 110 (2011) 043501-1.

[36] R. Zhai, H. Wang, H. Yan, J. Cryst. Growth 289 (2006) 647.

[37] F. Lei, B. Yan, J. Sol. State Chem. 181 (2008) 855.

[38] A. Golubovic, R. Gajic, Z. Dohcevic-Mitrovic, S. Nikolic, Sci. Sintering 38 (2006) 265.

[39] T. Thongtem, S. Kungwankunakorn, B. Kuntalue, A. Phuruangrat, S. Thongtem, J. Alloys Compnd. 506 (2010) 475.

[40] D. L. Wood, J. Tauc, Phys. Rev. B 5 (1972) 3144.

[41] R. Lacomba-Perales, J. Ruiz-Fuertes, D. Errandonea, D. Martínez-García, A. Segura, Eur. Phys. Lett. 83 (2008) 37002.

[42] V. M. Longo, E. Orhan, L. S. Cavalcante, S. L. Porto, J. W. M. Espinosa, J. A. Varela, E. Longo, Chem. Phys. 334 (2007) 180.

[43] J. C. Sczancoski, M. D. R. Bomio, L. S. Cavalcante, M. R. Joya, P. S. Pizani, J. A. Varela, E. Longo, L. M. Siu, J. A. Andrés, J. Phys. Chem. 113 (2009) 5812.

[44] J. H. Ryu, G. S. Park, K. M. Kim, C. S. Lim, J. Yoon, K. B. Shim, Appl. Phys. A88 (2007) 731.

[45] J. H. Ryu, S. Y. Bang, S. W. Kim, G. S. Park, K. M. Kim, J. Yoon, K. B. Shim, N. Koshizaki, J. Alloys Compnd. 441 (2007) 146.

[46] M. A. Maurera, A. G. Souza, L. E. B. Soledade, F. M. Pontes, E. Longo, E. R. Leite, J. A. Varela, Mater. Lett. 58 (2004) 727.

[47] K. J. Rao, B. Vaidhyanathan, M. Ganguli, P. A. Ramakrishnan, Chem. Mater. 11 (1999) 882.

[48] M. V. S. Lima, Diss. Mestrado, Programa de Pós Graduação em Química, UFSCar, S. Carlos, SP (2010).

(Rec. 16/09/2012, Rev. 12/02/2013, Ac. 13/02/2013) 